\title{
Relationship between In Vivo CYP3A4 Activity, CYP3A5 Genotype, and Systemic Tacrolimus Metabolite/Parent Drug Ratio in Renal Transplant Recipients and Healthy Volunteers ${ }^{\text {ฐ }}$
}

\author{
Thomas Vanhove, Hylke de Jonge, Henriëtte de Loor, Marlies Oorts, Jan de Hoon, \\ Anton Pohanka, Pieter Annaert, and Dirk R. J. Kuypers
}

Departments of Microbiology and Immunology (T.V., H.d.J., H.d.L., D.R.J.K.), Pharmaceutical and Pharmacological Sciences (J.d.H.), and Drug Delivery and Disposition, Department of Pharmaceutical and Pharmacological Sciences (M.O., P.A.), Catholic University of Leuven, Leuven, Belgium; Department of Nephrology and Renal Transplantation (T.V., H.d.J., H.d.L., D.R.J.K.), and Center for Clinical Pharmacology (J.d.H.), University Hospitals Leuven, Leuven, Belgium; Department of Laboratory Medicine, Division of Clinical Pharmacology, Karolinska Institutet, Stockholm, Sweden; (A.P.); and Department of Clinical Pharmacology, Karolinska University Hospital, Stockholm, Sweden (A.P.)

Received April 9, 2018; accepted August 28, 2018

\begin{abstract}
CYP3A5 genotype is a major determinant of tacrolimus clearance, and has been shown to affect systemic tacrolimus metabolite/parent ratios in healthy volunteers, which may have implications for efficacy and toxicity. In a cohort of 50 renal transplant recipients who underwent quantification of CYP3A4 activity using the oral midazolam drug probe, we confirmed that CYP3A5 genotype is the single most important determinant of tacrolimus metabolite/parent ratio [CYP3A5 expressors displayed 2.7- and 2-fold higher relative exposure to 13-desmethyltacrolimus (DMT) and 31-DMT, respectively; $P<0.001$ ].
\end{abstract}

There was, however, no relationship between CYP3A4 activity and tacrolimus metabolite/parent ratios. Additional analyses in 16 healthy volunteers showed that dual pharmacological inhibition of CYP3A4 and P-glycoprotein using itraconazole resulted in increased tacrolimus metabolite/parent ratios $(+65 \%,+112 \%$, and $25 \%$ for $13-, 15-$, and 31-DMT, respectively; $\boldsymbol{P}<0.01)$. This finding was confirmed in a cohort of nine renal transplant recipients who underwent tacrolimus pharmacokinetic assessments before and during CYP3A4 inhibition (58\% increase in overall metabolite/tacrolimus ratio; $P=0.017$ ).

\section{Introduction}

Tacrolimus, a calcineurin inhibitor used to prevent rejection in solid organ transplantation, is a dual substrate for CYP3A4 and CYP3A5 (collectively referred to as CYP3A) and the P-glycoprotein (P-gp) efflux pump [encoded by the ATP-binding cassette subfamily $\mathrm{B}$ member $1(A B C B 1)$ gene]. CYP3A and P-gp are present in enterocytes (limiting intestinal uptake) and hepatocytes (contributing to first-pass metabolism and systemic clearance). The vast majority of systemically absorbed tacrolimus undergoes hepatic biotransformation to metabolites that are excreted in the feces, while renal metabolism and excretion account for less than $5 \%$ of parent compound (Möller et al., 1999). Between- and within-subject differences in the activity of CYP3A (and probably of P-gp) contribute significantly to tacrolimus' high pharmacokinetic and pharmacodynamic variability (Vanhove et al., 2016). CYP3A5 expression and activity in intestine, liver, kidney, and other organs are strongly reduced in carriers of two loss-of-function $C Y P 3 A 5$ alleles $(C Y P 3 A 5 * 3$, $* 6$, or *7). In these CYP3A5 nonexpressors, tacrolimus apparent oral clearance $(\mathrm{CL} / \mathrm{F})$ is about 2.5 -fold lower compared with carriers of at least one active $C Y P 3 A 5 * 1$ allele (CYP3A5 expressors) (de Jonge et al., 2013). The effect of $C Y P 3 A 5$ genotype is not limited to an increased rate of metabolic clearance. As Zheng et al. (2012) have demonstrated,

https://doi.org/10.1124/dmd.118.081935.

S This article has supplemental material available at dmd.aspetjournals.org.
CYP3A5 genotype also strongly influences relative systemic exposure to tacrolimus metabolites and likely influences local tissue concentrations of tacrolimus (and its metabolites). In a study in 24 healthy volunteers, systemic metabolite/parent area under the curve (AUC) ratios for the primary metabolites $13-O$-desmethyl tacrolimus (DMT) M1, 31-DMT M2, and 12-hydroxy tacrolimus M4 were 2.0- to 2.7-fold higher in CYP3A5 expressors (Zheng et al., 2012). Furthermore, a semiphysiological model predicted $47 \%$ lower tacrolimus exposure in renal epithelium (normalized to blood AUC) in CYP3A5 expressors compared with CYP3A5 nonexpressors. The fact that systemic and intrarenal exposure to tacrolimus and its primary metabolites is related to CYP3A5 genotype could have pharmacodynamic (i.e., efficacy) and toxicodynamic implications. It has, for example, been shown that the immunosuppressive activity of 31-DMT is comparable to that of tacrolimus (Iwasaki et al., 1995). Whether tacrolimus metabolites are nephrotoxic has not been studied, although the nephrotoxicity of several metabolites of the related calcineurin inhibitor ciclosporin is well established in vitro and in animal models; some evidence suggests it may also be relevant in humans (Copeland et al., 1990; Radeke et al., 1991; Stephens et al., 2006).

It is unclear to what extent tacrolimus metabolite/parent ratio varies in a relevant population of renal transplant recipients and how much CYP3A5 genotype contributes to interindividual variability in metabolite exposure in such a population. In particular, since the allelic frequency of $C Y P 3 A 5 * 1$ is only $5 \%-15 \%$ in Caucasians (Lamba et al., 2012),

ABBREVIATIONS: AUC, area under the curve; CL/F, apparent oral clearance; DMT, desmethyl tacrolimus; HOMDZ, hydroxy midazolam; MDZ, midazolam; P-gp, P-glycoprotein. 
variability in other determinants of tacrolimus metabolism, namely, the activity of CYP3A4, may also contribute to differences in relative metabolite exposure. We have previously shown that in vivo CYP3A4 activity, assessed using the oral midazolam (MDZ) probe, explains up to $30 \%$ of interindividual variability in steady-state tacrolimus clearance (de Jonge et al., 2012). Therefore, the goals of this study were 1) to determine the degree of interindivual variability in tacrolimus metabolite/parent ratio in a population of renal transplant recipients, 2) to analyze whether this variability can be related to differences in CYP3A4 activity, and 3) to assess whether combined pharmacological inhibition of CYP3A4 and $\mathrm{P}$-gp can be used to influence tacrolimus metabolite/parent ratio.

\section{Materials and Methods}

Study Populations. Two cohorts were analyzed. The principal cohort (A) comprised 50 renal allograft recipients in tacrolimus steady state, in which $\mathrm{AUC}_{0-8}$ profiles of midazolam, tacrolimus, and their primary metabolites were determined. The goal of this analysis was to determine whether interindividual differences in CYP3A4 activity are related to interindividual differences in tacrolimus metabolite/parent ratio. Cohort B addressed the question of whether tacrolimus metabolite/parent ratio can be influenced by pharmacological inhibition of CYP3A4 activity. In this study, 16 male healthy volunteers underwent $\mathrm{AUC}_{0-24}$ profiles for MDZ and (single-dose) tacrolimus before and immediately after administration of the potent CYP3A4/P-gp inhibitor itraconazole. Methodological details for both cohorts are provided subsequently.

Cohort A was a subgroup of patients from a previously reported prospective single center study (Vanhove et al., 2017b). Stable renal allograft recipients $\geq 1$ year post-transplant treated with once-daily tacrolimus (Advagraf; Astellas Pharma Europe, Staines, United Kingdom) were asked to participate. Exclusion criteria were age less than 18 years; combined organ transplants (except kidney/pancreas); women of childbearing potential not using acceptable contraceptive measures; pregnant women, hemoglobin $<8 \mathrm{~g} / \mathrm{dl}$; albumin $<25 \mathrm{~g} / \mathrm{l}$; intestinal malabsorption; liver cirrhosis; alanine aminotransferase or bilirubin $>2 \times$ upper limit of normal; estimated glomerular filtration rate $<15 \mathrm{ml} / \mathrm{min}$, calculated from serum creatinine using the modification of diet in renal disease equation (Levey et al., 1993); change in tacrolimus dose in the 3 days prior to the study; documented noncompliance; addiction to any known drug or alcohol; known allergy or intolerance to MDZ or fexofenadine; and use of a moderate or potent CYP3A4 inhibitor or inducer. Patients were asked to abstain from alcohol use 1 week prior to the study and from any fruit juice, grapefruit, or pomelo 3 days prior to the study. The details of the pharmacokinetic study protocol have been previously described (Vanhove et al., 2017b). Briefly, patients presented at the outpatient clinic after an overnight fast. At 8:00 AM, patients were orally administered $2 \mathrm{mg}$ of MDZ ( $2 \mathrm{ml}$ of a $1 \mathrm{mg} / \mathrm{ml} \mathrm{MDZ} \mathrm{solution,} \mathrm{Dormicum;} \mathrm{Roche,} \mathrm{Basel,} \mathrm{Switzerland)} \mathrm{in} 30 \mathrm{ml}$ of a $5 \%$ glucose solution and their usual dose of tacrolimus and other immunosuppressive medication, followed by $100 \mathrm{ml}$ of water to rinse the glass. All other medication was ingested at 10:00 AM, followed by a standard breakfast. Two 4-ml EDTA blood tubes were collected immediately before and at $0.25,0.5,1,1.5,2,3,4,5,6$, and 8 hours after probe drug administration.

Cohort B was a prospective single-arm open-label study. Sixteen healthy adult nonsmoking males with no significant medical history were recruited. None of the subjects had taken any CYP3A4/5 interfering medication for at least 1 month prior to the start of and during the study. All subjects abstained from alcohol, grapefruit, or pomelo from 7 days prior to the start of the study until study conclusion. On days 1 and 6, subjects were administered a single oral dose of $3 \mathrm{mg}$ tacrolimus (Advagraf; Astellas Pharma Europe) and $2 \mathrm{mg}$ of MDZ (2 $\mathrm{ml}$ of a $1 \mathrm{mg} / \mathrm{ml}$ solution of Dormicum; Roche) with $250 \mathrm{ml}$ of water in the morning after an overnight fast. Two 4-ml blood samples were collected in ethylenediaminetetraacetic acid-containing tubes before and at $0.5,1,1.5,2,3,4,5,6,8,10,12$, and 24 hours after drug administration. One whole blood sample was stored at $-80^{\circ} \mathrm{C}$ pending tacrolimus metabolite quantification. The other sample was centrifuged for 10 minutes at $1860 \mathrm{~g}$ and $4^{\circ} \mathrm{C}$, after which plasma was stored at $-80^{\circ} \mathrm{C}$ pending MDZ quantification. On days 2-5, $200 \mathrm{mg}$ of itraconazole (two tablets of $100 \mathrm{mg}$ Sporanox; Janssen-Cilag, Beerse, Belgium) was administered twice daily with $250 \mathrm{ml}$ of water, 2 hours before breakfast/dinner.

All parts of this study were performed according to the latest version of the Declaration of Helsinki and were approved by the ethics committee of University
Hospitals Leuven (ML5159; S51157; S58603) and the Belgian Federal Agency for Medicines and Health Products (EudraCT 2008-004158-33 and 2015-00451874, https://eudract.ema.europa.eu). All participants provided written informed consent.

Analytical Methods. Plasma concentrations of MDZ and its major metabolites 1'-hydroxy midazolam (HOMDZ) and 4-HOMDZ were measured using a highperformance liquid chromatography-tandem mass spectrometry method, as previously described (de Loor et al., 2011). Whole blood concentrations of tacrolimus and its major metabolites 13-DMT, 15-DMT, and 31-DMT were measured using an ultraperformance liquid chromatography-tandem mass spectrometry method, as described in the Supplemental Material. Tacrolimus and its metabolites form ionic adducts with either ammonium or sodium. Only the most abundant adduct, and which was the most stable across measurements, was used for analysis; for tacrolimus, 15-DMT, and 31-DMT, this was the ammonium adduct, and for 13-DMT, this was the sodium adduct.

Genotyping. Genomic DNA was isolated from whole blood samples using a salting out procedure (Miller et al., 1988). The quantity and quality of genomic DNA were verified with a NanoDrop spectrophotometer (Thermo Fisher Scientific, Waltham, MA) before being assayed on an OpenArray platform (Life Technologies, Carlsbad, CA). Participants were genotyped for the following single nucleotide polymorphisms, among others: CYP3A5*1 (rs776746); CYP3A4*22 (rs35599367); and ABCB1 3435C $>\mathrm{T}$ (rs 1045642), 2677G $>\mathrm{T} / \mathrm{A}$ (rs2032582), and $1236 \mathrm{C}>\mathrm{T}$ (rs1128503). Hardy-Weinberg equilibrium and linkage disequilibrium between single nucleotide polymorphisms were assessed using Haploview (Barrett et al., 2005). Haplotypes were inferred using the program PHASE version 2.1 (Stephens et al., 2001). ABCB1 single nucleotide polymorphisms were grouped into four diplotype categories based on the presence of wild-type CGC and triple mutated (presumed loss-of-function) TTT haplotypes: CGC-CGC, CGC-TTT, TTTTTT, and others (containing one or more other haplotypes) (Vanhove et al., 2017a).

Pharmacokinetic and Statistical Analysis. Data are presented as mean \pm S.D. except when stated otherwise. Normality was tested using the Shapiro-Wilk test. $\mathrm{AUC}, \mathrm{CL} / \mathrm{F}, C_{\max }$, and $\mathrm{AUC}_{\text {metabolite }} / \mathrm{AUC}_{\text {tacrolimus }}$ values were not normally distributed and natural logarithm (Ln) transformed for analysis. Differences between mean values were assessed using an independent samples $t$ test for normally distributed continuous data (analysis of variance in the case of more than two categories of the predictor variable) and the Mann-Whitney $U$ test for ordinal data. Since steady-state exposure to tacrolimus and its primary metabolites was the outcome measure of interest, no compartmental analysis was performed. Independent predictors of tacrolimus metabolite/parent AUC ratio were determined using multivariable linear regression. The following variables were entered as possible predictors of metabolite/parent ratio: age, time after transplantation, hematocrit, actual body weight, presence of diabetes mellitus, CYP3A5 genotype, $A B C B 1$ functional diplotype group, MDZ CL/F, AUC 1'-HOMDZ $^{\prime}$ $\mathrm{AUC}_{\mathrm{MDZ}}$ ratio, and tacrolimus $\mathrm{CL} / \mathrm{F}$ (the latter not included in the same model as MDZ CL/F), followed by stepwise removal of nonsignificant predictor variables. A two-sided $P$ value of $<0.05$ was considered statistically significant. All reported $R^{2}$ values are semipartial. Statistical analyses were performed using IBM SPSS Statistics version 22 (IBM, New York, NY). Calculation of noncompartmental analysis pharmacokinetic parameters and figure generation were performed using Graphpad Prism version 6 (Graphpad Prism, La Jolla, CA).

\section{Results}

Tacrolimus Metabolite/Parent Ratio Is Related to CYP3A5 Genotype, but Not to Baseline CYP3A4 Activity. First, the relationship between CYP3A5 genotype, CYP3A4 activity, and systemic tacrolimus metabolite/parent ratio was examined in a cohort of 50 tacrolimus-treated renal recipients under steady-state conditions (cohort A). Characteristics of cohort A are summarized in Supplemental Table 1 . The $C Y P 3 A 5^{*} 1$ allele was present in 10 renal recipients (i.e., $20 \%$ expressors). One CYP3A5 nonexpressor carried the CYP3A4*22 allele. Noncompartmental analysis pharmacokinetic parameters of MDZ, tacrolimus, and their primary metabolites are presented in Table 1. Mean concentration-time profiles are shown in Fig. 1.

MDZ CL/F did not differ between CYP3A5 expressors and nonexpressors, in agreement with previous reports (Kharasch et al., 2007; de Jonge et al., 2013). As expected, mean tacrolimus CL/F was 
TABLE 1

Pharmacokinetic parameters of midazolam, tacrolimus, and their primary metabolites in cohort A, stratified by CYP3A5 genotype

\begin{tabular}{|c|c|c|c|c|}
\hline Variable & Nonexpressors $(n=40)$ & Expressors $(n=10)$ & Difference & $P$ \\
\hline & & & $\%$ & \\
\hline \multicolumn{5}{|l|}{ Midazolam } \\
\hline $\mathrm{AUC}_{0-8}(\mathrm{~h} \cdot \mathrm{ng} / \mathrm{ml})$ & $3373 \pm 1764$ & $3355 \pm 1219$ & & 0.770 \\
\hline$T_{\max }(\mathrm{h})^{a}$ & $0.5(0.3-0.5)$ & $0.5(0.5-1.0)$ & & 0.519 \\
\hline$C_{\max }(\mathrm{ng} / \mathrm{ml})$ & $16.8 \pm 8.1$ & $15.9 \pm 7.2$ & -5.4 & 0.811 \\
\hline $\mathrm{CL} / \mathrm{F}(\mathrm{ml} / \mathrm{h})$ & $735 \pm 324$ & $673 \pm 249$ & -8.4 & 0.770 \\
\hline \multicolumn{5}{|l|}{ Midazolam metabolites } \\
\hline $\mathrm{AUC}_{1^{\prime}-\mathrm{HOMDZ}} / \mathrm{AUC}_{\mathrm{MDZ}}$ & $0.43 \pm 0.22$ & $0.39 \pm 0.17$ & -9.3 & 0.544 \\
\hline $\mathrm{AUC}_{4-\mathrm{HOMDZ}} / \mathrm{AUC}_{\mathrm{MDZ}}$ & $0.05 \pm 0.01$ & $0.04 \pm 0.01$ & -20.0 & 0.108 \\
\hline \multicolumn{5}{|l|}{ Tacrolimus } \\
\hline $\mathrm{AUC}_{0-24}(\mathrm{~h} \cdot \mathrm{ng} / \mathrm{ml})$ & $266.0 \pm 66.3$ & $291.8 \pm 59.2$ & +9.7 & 0.257 \\
\hline Dose (mg/day) & $4.7 \pm 2.2$ & $10.5 \pm 3.1$ & +123.4 & $<0.001$ \\
\hline$T_{\max }(\mathrm{h})^{a}$ & $2.0(1.6-3.0)$ & $1.75(1.0-3.0)$ & & 1.000 \\
\hline$C_{\max }(\mathrm{ng} / \mathrm{ml})$ & $17.9 \pm 5.0$ & $25.6 \pm 7.3$ & +43.0 & 0.002 \\
\hline $\mathrm{CL} / \mathrm{F}(\mathrm{L} / \mathrm{h})$ & $17.8 \pm 7.6$ & $35.6 \pm 8.2$ & +100.0 & $<0.001$ \\
\hline \multicolumn{5}{|l|}{ Tacrolimus metabolites } \\
\hline $\mathrm{AUC}_{13-\mathrm{DMT}} / \mathrm{AUC}_{\text {tacrolimus }}$ & $0.036 \pm 0.012$ & $0.100 \pm 0.024$ & +177.8 & $<0.001$ \\
\hline $\mathrm{AUC}_{15-\mathrm{DMT}} / \mathrm{AUC}_{\text {tacrolimus }}$ & $0.031 \pm 0.007$ & $0.031 \pm 0.007$ & & 0.814 \\
\hline $\mathrm{AUC}_{31-\mathrm{DMT}} / \mathrm{AUC}_{\text {tacrolimus }}$ & $0.003 \pm 0.001$ & $0.006 \pm 0.001$ & +100.0 & $<0.001$ \\
\hline $\mathrm{AUC}_{\text {metabolites }} / \mathrm{AUC}_{\text {tacrolimus }}$ & $0.070 \pm 0.017$ & $0.136 \pm 0.028$ & +94.3 & $<0.001$ \\
\hline
\end{tabular}

$\mathrm{AUC}_{\text {metabolites}}$, sum of AUC for 13-DMT, 15-DMT, and 31-DMT; $T_{\max }$, time to reach maximum blood concentration. ${ }^{a}$ Median (interquartile range).

2-fold higher in CYP3A5 expressors, whose tacrolimus AUC was similar to that of nonexpressors despite a daily tacrolimus dose that was 2.2-fold higher on average. Independent predictors of tacrolimus $\mathrm{CL} / \mathrm{F}$ in multivariable regression were CYP3A5 genotype $(\mathrm{B}=0.67$, $\left.R^{2}=0.349, P<0.001\right), \mathrm{MDZ} \mathrm{CL} / \mathrm{F}\left(\mathrm{B}=0.55, R^{2}=0.223, P<0.001\right)$, hematocrit $\left(\mathrm{B}=-3.40, R^{2}=0.061, P=0.001\right)$, and weight $(\mathrm{B}=0.01$, $R^{2}=0.036, P=0.019$; overall $\left.R^{2}=0.669\right)$. When limiting the analysis to CYP3A5 nonexpressors, predictors of tacrolimus CL/F were MDZ $\mathrm{CL} / \mathrm{F}\left(\mathrm{B}=0.65, R^{2}=0.433, P<0.001\right)$ and hematocrit $(\mathrm{B}=-3.14$, $R^{2}=0.100, P=0.005$; overall $\left.R^{2}=0.533\right)$. In CYP3A5 expressors, the only predictor of tacrolimus $\mathrm{CL} / \mathrm{F}$ was weight $\left(\mathrm{B}=0.009, R^{2}=0.435\right.$, $P=0.023)$
Relative systemic exposure to 13-DMT and 31-DMT, measured using $\mathrm{AUC}_{\text {metabolite }} / \mathrm{AUC}_{\text {tacrolimus, was }} 178 \%$ and $100 \%$ higher in CYP3A5 expressors compared with CYP3A5 nonexpressors $(P<$ 0.001); $\mathrm{AUC}_{15-\mathrm{DMT}} / \mathrm{AUC}_{\text {tacrolimus }}$ did not differ between CYP3A5 expressors and nonexpressors. The overall result was a 94\% higher $\mathrm{AUC}_{\text {metabolites }} / \mathrm{AUC}_{\text {tacrolimus }}$ ratio in CYP3A5 expressors $(P<0.001)$. Within CYP3A5 nonexpressors, the range of observed metabolite/parent ratios was relatively narrow (range $1.9 \%-6.7 \%$ for $\mathrm{AUC}_{13-\mathrm{DMT}}$ / $\mathrm{AUC}_{\text {tacrolimus }}$ and $0.1 \%-0.5 \%$ for $\mathrm{AUC}_{31-\mathrm{DMT}} / \mathrm{AUC}_{\text {tacrolimus }}$, although it was $0.2 \%-5.0 \%$ for $\left.\mathrm{AUC}_{15-\mathrm{DMT}} / \mathrm{AUC}_{\text {tacrolimus }}\right)$. Tacrolimus metabolite/parent ratios did not differ between $A B C B 1$ functional diplotype categories ( $P \geq 0.4$ for all analyses).
CYP3A5 non-expressors $(n=40)$

A

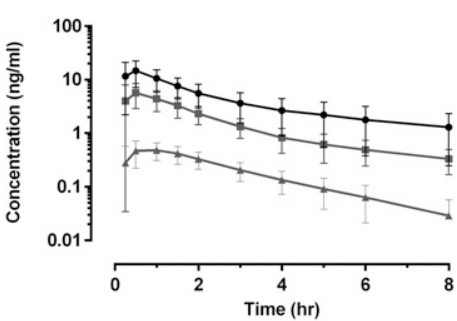

C



CYP3A5 expressors $(n=10)$

B

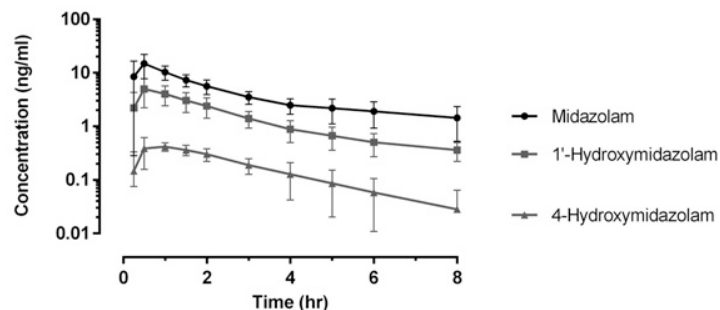

D

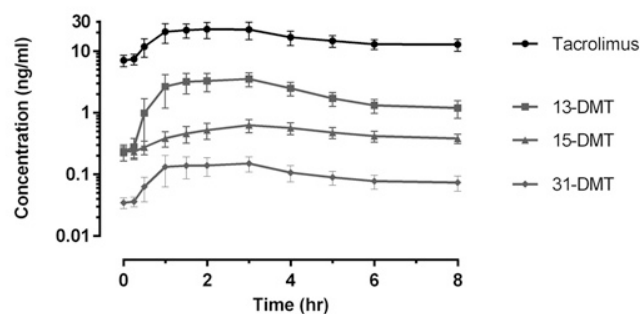

Fig. 1. Mean $\log$ concentration-time profiles of midazolam, tacrolimus, and their primary metabolites in cohort A. The left-hand panels display CYP3A5 non-expressors (B and D) and the right-hand panels display CYP3A5 expressors (A and C). Error bars represent the S.D. 
A

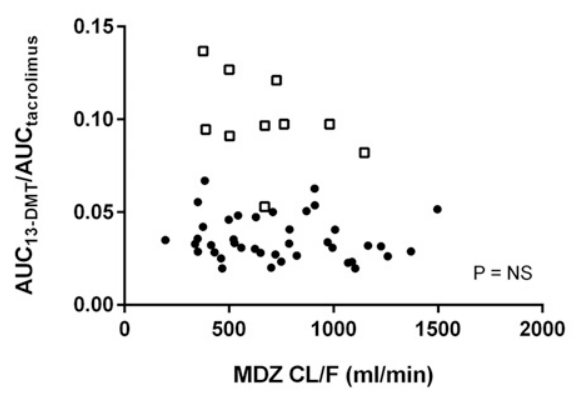

C

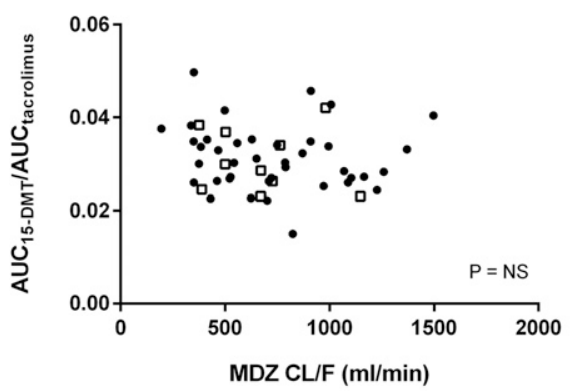

E

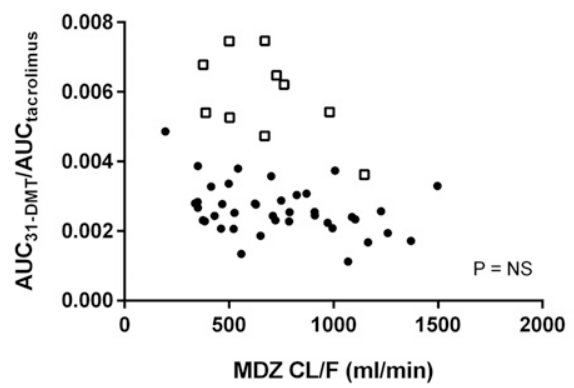

B

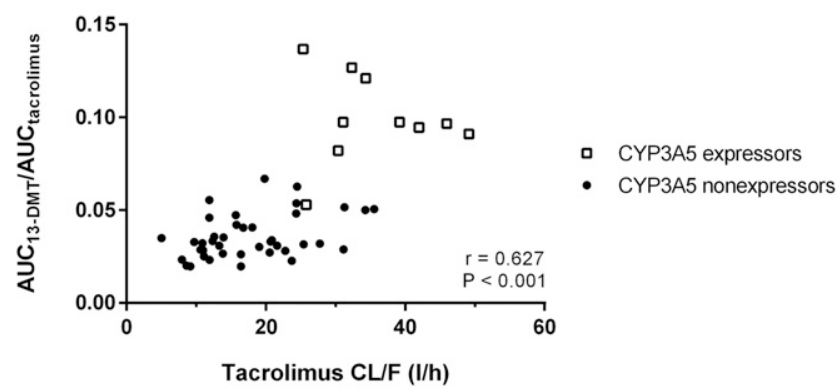

D

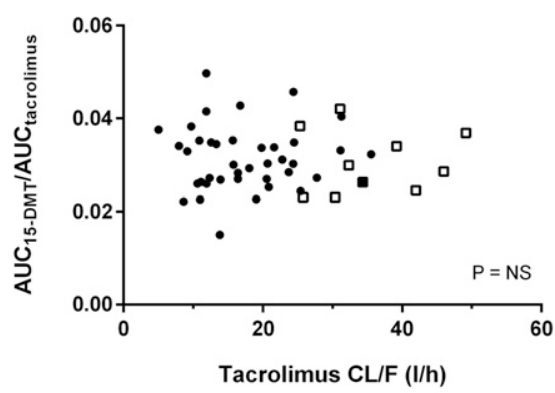

$\mathbf{F}$

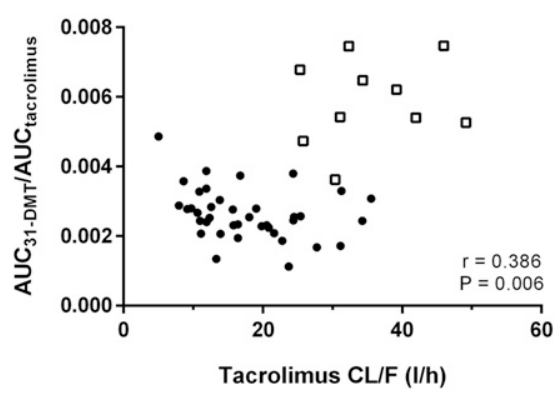


and F) in cohort $\mathrm{A}$.

There was a consistently negative correlation between MDZ CL/F and all tacrolimus metabolite/parent ratios, but this only reached statistical significance for $\mathrm{AUC}_{31-\mathrm{DMT}} / \mathrm{AUC}_{\text {tacrolimus }}$ in the subgroup of CYP3A5 nonexpressors $(R=-0.38, P=0.015)$. No significant correlation was observed between $\mathrm{MDZ} \mathrm{CL} / \mathrm{F}$ and $\mathrm{AUC}_{13-\mathrm{DMT}} / \mathrm{AUC}_{\text {tacrolimus }}$ or $\mathrm{AUC}_{15-\mathrm{DMT}} / \mathrm{AUC}_{\text {tacrolimus }}$ in either CYPA5 nonexpressors or expressors, as shown in Fig. 2 and Supplemental Table 2. Similarly, there was no significant correlation between MDZ metabolite/parent ratios $\left(\mathrm{AUC}_{1^{\prime}-\mathrm{HOMDZ}} / \mathrm{AUC}_{\mathrm{MDZ}}\right.$ or $\left.\mathrm{AUC}_{4-\mathrm{HOMDZ}} / \mathrm{AUC}_{\mathrm{MDZ}}\right)$ and the different $\mathrm{AUC}_{\text {metabolite }} / \mathrm{AUC}_{\text {tacrolimus }}$ ratios (data not shown). Tacrolimus CL/F was moderately correlated with $\mathrm{AUC}_{13-\mathrm{DMT}} / \mathrm{AUC}_{\text {tacrolimus }}(r=0.627$, $P<0.001)$ and $\mathrm{AUC}_{31-\mathrm{DMT}} / \mathrm{AUC}_{\text {tacrolimus }}(r=0.386, P=0.006)$, indicating that relative exposure to $13-\mathrm{DMT}$ and 31 -DMT increased in parallel with higher tacrolimus clearance (Fig. 2). This effect was largely driven by high tacrolimus CL/F values in the CYP3A5 expressors. In the subgroup of CYP3A5 nonexpressors, the association between $\mathrm{AUC}_{13-\mathrm{DMT}} / \mathrm{AUC}_{\text {tacrolimus }}$ and tacrolimus $\mathrm{CL} / \mathrm{F}$ was weaker $(r=0.397$, $P=0.011)$ and that between $\mathrm{AUC}_{31-\mathrm{DMT}} / \mathrm{AUC}_{\text {tacrolimus }}$ was reversed ( $r=-0.384, P=0.014)$. In multivariable analysis, CYP3A5 genotype was the dominant determinant of $\mathrm{AUC}_{13-\mathrm{DMT}} / \mathrm{AUC}_{\text {tacrolimus }}$ and $\mathrm{AUC}_{31-\mathrm{DMT}} /$


variability, respectively (see Supplemental Table 3).
Itraconazole Increases the Tacrolimus Metabolite/Parent Ratio. Next, we studied whether pharmacological CYP3A4/P-gp inhibition alters the tacrolimus metabolite/parent ratio in a cohort of 16 healthy volunteers treated with itraconazole (cohort B). Characteristics of cohort $B$ are presented in Supplemental Table 4. Pharmacokinetic parameters of tacrolimus and its three primary metabolites before and during CYP3A4 inhibition are shown in Table 2; mean blood concentration-time profiles are presented in Fig. 3. Metabolite/parent ratios for 13-DMT and 31-DMT were higher in CYP3A5-expressing healthy volunteers (see Supplemental Table 5), analogous to the renal transplant recipients in cohort A. Mean tacrolimus $\mathrm{AUC}_{0-24}$ increased 3.3-fold as a result of itraconazole treatment. Metabolite/parent ratios for all three metabolites were significantly increased during CYP3A4/P-gp inhibition (plotted in Fig. 4). This increase in relative exposure averaged 1.7-, 2.1-, 1.3-, and 1.8-fold for 13-DMT, 15-DMT, 31-DMT, and all three metabolites combined, respectively $(P<0.01$ for all). There was a numerically smaller increase in $\mathrm{AUC}_{15-\mathrm{DMT}} / \mathrm{AUC}_{\text {tacrolimus }}$ resulting from itraconazole treatment in CYP3A5 expressors versus CYP3A5 nonexpressors, but this difference was not statistically significant $(68 \% \pm 30 \%$ vs. $132 \% \pm 66 \%, P=0.077)$. Similarly, there was no difference between CYP3A5 expressors and nonexpressors with regard to changes in tacrolimus $\operatorname{AUC}(P=0.944), \mathrm{AUC}_{13-\mathrm{DMT}} / \mathrm{AUC}_{\text {tacrolimus }}(P=0.353)$, or 
TABLE 2

Pharmacokinetic parameters of tacrolimus and its primary metabolites before and during CYP3A4 inhibition in 16 healthy volunteers (cohort B)

\begin{tabular}{|c|c|c|c|c|}
\hline Variable & Baseline & CYP3A4 Inhibition & Difference & $P$ \\
\hline & & & $\%$ & \\
\hline \multicolumn{5}{|l|}{ Tacrolimus } \\
\hline $\mathrm{AUC}_{0-24}(\mathrm{~h} \cdot \mathrm{ng} / \mathrm{ml})$ & $50.6 \pm 21.6$ & $167.9 \pm 73.2$ & +232 & $<0.001$ \\
\hline $\mathrm{CL} / \mathrm{F}(1 / \mathrm{h})$ & $69.7 \pm 28.9$ & $21.4 \pm 9.3$ & -69 & $<0.001$ \\
\hline \multicolumn{5}{|l|}{ Midazolam } \\
\hline $\mathrm{CL} / \mathrm{F}(\mathrm{ml} / \mathrm{min})$ & $762.7 \pm 327.7$ & $83.4 \pm 24.9$ & -89.1 & $<0.001$ \\
\hline $\mathrm{AUC}_{1^{\prime}-\mathrm{HOMDZ}} / \mathrm{AUC}_{\mathrm{MDZ}}$ & $0.434 \pm 0.133$ & $0.041 \pm 0.013$ & -90.6 & $<0.001$ \\
\hline $\mathrm{AUC}_{4-\mathrm{HOMDZ}} / \mathrm{AUC}_{\mathrm{MDZ}}$ & $0.081 \pm 0.017$ & $0.030 \pm 0.009$ & -63.0 & $<0.001$ \\
\hline \multicolumn{5}{|l|}{ Tacrolimus metabolites } \\
\hline $\mathrm{AUC}_{13-\mathrm{DMT}} / \mathrm{AUC}_{\text {tacrolimus }}$ & $0.065 \pm 0.028$ & $0.107 \pm 0.038$ & +65 & $<0.001$ \\
\hline $\mathrm{AUC}_{15-\mathrm{DMT}} / \mathrm{AUC}_{\text {tacrolimus }}$ & $0.025 \pm 0.006$ & $0.053 \pm 0.014$ & +112 & $<0.001$ \\
\hline $\mathrm{AUC}_{31-\mathrm{DMT}} / \mathrm{AUC}_{\text {tacrolimus }}$ & $0.004 \pm 0.002$ & $0.005 \pm 0.002$ & +25 & 0.007 \\
\hline $\mathrm{AUC}_{\text {metabolites }} / \mathrm{AUC}_{\text {tacrolimus }}$ & $0.094 \pm 0.030$ & $0.166 \pm 0.040$ & +77 & $<0.001$ \\
\hline
\end{tabular}

$\mathrm{AUC}_{\text {metabolites }}$, sum of AUC for 13-DMT, 15-DMT, and 31-DMT.

$\mathrm{AUC}_{31-\mathrm{DMT}} / \mathrm{AUC}_{\text {tacrolimus }}(P=0.878)$. Baseline $\mathrm{AUC}_{\text {metabolites }} / \mathrm{AUC}_{\text {tacrolimus }}$ was strongly correlated to the degree of change (delta) in $\mathrm{AUC}_{\text {metabolites }}$ ' $\mathrm{AUC}_{\text {tacrolimus }}$ resulting from itraconazole inhibition $(R=0.67, P=0.005$; see Fig. 5).

Contrary to tacrolimus, relative exposure to $\mathrm{MDZ}$ metabolites decreased as a result of CYP3A4 inhibition. $\mathrm{AUC}_{1^{\prime}-\mathrm{HOMDZ}} / \mathrm{AUC}_{\mathrm{MDZ}}$ was $91 \%$ lower during CYP3A4 inhibition (see Table 2). No adverse events occurred in any of the study cohorts; no subjects withdrew from the study. Because cohort B differs significantly from a clinical setting (in particular, lack of steady state resulting from administration of single doses of tacrolimus), these analyses were repeated on a cohort of nine renal transplant recipients cotreated with tacrolimus and a variety of moderate-to-potent CYP3A4 inhibitors for clinical reasons (cohort C), detailed in the Supplemental Material.

\section{Discussion}

In an analysis of 50 tacrolimus-treated renal transplant recipients in steady state (cohort A), CYP3A5 genotype was the single most important determinant of systemic tacrolimus metabolite/parent ratio. Compared with CYP3A5 nonexpressors, CYP3A5 expressors displayed 2.7- and 2-fold higher relative exposure to 13-DMT and 31-DMT, respectively, while relative exposure to 15 -DMT was identical. These findings are in close agreement with the observations made by Zheng et al. (2012) in 24 healthy volunteers administered a single dose of tacrolimus. In the current population of renal transplant recipients in steady state, interindividual variability in tacrolimus metabolite/parent ratios was relatively limited. Furthermore, around $60 \%$ of interindividual variability in the metabolite/parent ratio of 13-DMT and 31-DMT was explained by CYP3A5 genotype, with only minimal overlap between CYP3A5 expressors and nonexpressors (see Fig. 2).
To our knowledge, this is the first study to investigate the relationship between in vivo combined intestinal and hepatic CYP3A4 activity, assessed using the oral MDZ probe, and relative systemic exposure to the metabolites of a CYP3A4 substrate such as tacrolimus. MDZ CL/F varied considerably (more than 6-fold) between renal transplant recipients and explained $43 \%$ of interindividual variability in tacrolimus $\mathrm{CL} / \mathrm{F}$ in CYP3A5 nonexpressors, in line with previous observations (de Jonge et al., 2012) and confirming that variability in CYP3A4 activity is a major determinant of variability in tacrolimus CL/F. However, this baseline CYP3A4 activity bore little relationship to tacrolimus metabolite/parent ratios. MDZ CL/F was negatively correlated with metabolite/parent ratios, but this was only statistically significant in CYP3A5 nonexpressors for 31-DMT, a quantitatively minor metabolite with concentrations 10- to 20-fold lower than those of 13-DMT and 15-DMT. To further elaborate on this relationship, analyses were performed in healthy volunteers (cohort B), demonstrating that administration of a dual CYP3A4/P-gp inhibitor significantly increased metabolite/parent ratios of all three primary tacrolimus metabolites. This observation suggests that itraconazole has a more pronounced impact on the clearance of the metabolites than on the clearance of tacrolimus.

The underlying mechanisms for the increased metabolite/parent ratios, both in CYP3A5 expressors and after CYP3A4/P-gp inhibition, remain speculative. In CYP3A5 expressors the significantly higher metabolite/parent ratios for 13-DMT and 31-DMT indicate that enhanced formation of these metabolites is not compensated for by a proportional increase in their elimination rates. This implies that 13-DMT and 31-DMT display nonlinear pharmacokinetic behavior, or a proportional decrease in clearance (i.e., elimination capacity) with increasing metabolite levels, possibly related to different affinities of tacrolimus and its metabolites for the catalytic site of CYP3A5. This may be especially relevant for CYP3A5, since it is a


Fig. 3. Mean log concentration-time profiles of tacrolimus and its primary metabolites in cohort $B$ (healthy volunteers), before and immediately after inhibition of CYP3A4 using itraconazole. Error bars represent the S.D. 

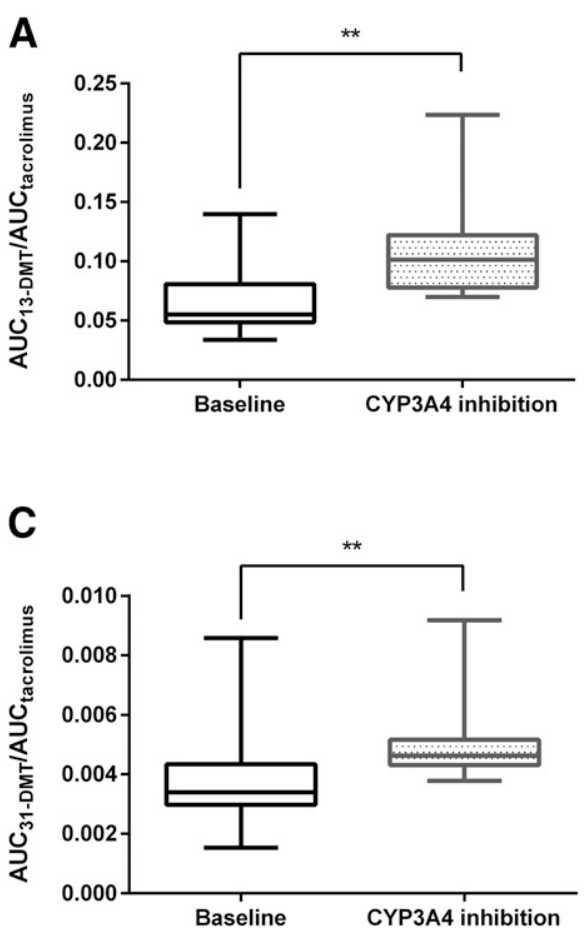


Fig. 4. (A-D) Tacrolimus metabolite/parent ratios during baseline and CYP3A4 inhibited conditions in cohort B (healthy volunteers). $* * P<0.01$ much less abundant enzyme compared with CYP3A4, and therefore is more susceptible to enzymatic saturation, particularly when parent and metabolites are competing. It is also possible that partial saturation of biliary excretion of metabolites occurs when metabolite generation rate is increased (i.e., in CYP3A5 expressors). Indeed, tacrolimus is almost exclusively eliminated through biliary excretion of its metabolites (rather than the unchanged parent molecule) (Möller et al., 1999). The observations that biliary concentrations of primary tacrolimus metabolites are significantly higher than those of tacrolimus (Shimomura et al., 2008) and that cholestasis results in accumulation of second-order metabolites (Gonschior et al., 1996) suggest that (P-gp-mediated) biliary excretion of primary metabolites may contribute more to their clearance than further metabolism to second- and third-order metabolites. If P-gp-mediated biliary excretion was to be the rate-limiting step in tacrolimus metabolite elimination and a source of nonlinear pharmacokinetic behavior, this would also imply that the increase in metabolite/parent ratio

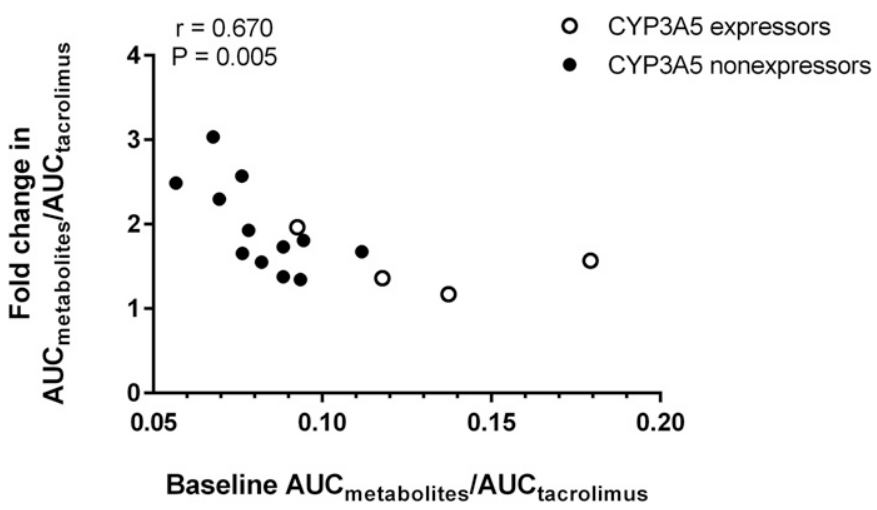

Fig. 5. Correlation between baseline $A U C_{\text {metabolites }} / A U C_{\text {tacrolimus }}$ and fold change (delta) $\mathrm{AUC}_{\text {metabolites }} / \mathrm{AUC}_{\text {tacrolimus }}$ resulting from itraconazole inhibition in cohort $\mathrm{B}$ (healthy volunteers). resulting from itraconazole administration may primarily be related to the P-gp-inhibiting properties of itraconazole and its metabolites (Wang et al., 2002; Vermeer et al., 2016), rather than its CYP3A4-inhibiting effects. Indeed, the fact that itraconazole reduced $\mathrm{AUC}_{1^{\prime}-\mathrm{HOMDZ}} / \mathrm{AUC}_{\mathrm{MDZ}}$ indicates that, for a drug that is no P-gp (or hepatic transporter) substrate, CYP3A4 inhibition results in less metabolite formation, as is to be expected. It is clear, however, that these are only hypotheses, the verification of which would require additional (in vitro) experiments.

Some limitations must be noted. First, urine collections were not available, thus apparent urinary tacrolimus clearances could not be calculated. Second, only 8-hour AUCs were available for renal transplant recipients (cohort A). However, because these were performed under steady-state pharmacokinetic conditions, it is reasonable to assume that tacrolimus $\mathrm{C}_{0}=\mathrm{C}_{24}$. Third, 12-hydroxy tacrolimus was not measured, although this is a quantitatively minor metabolite (Zheng et al., 2012).

In conclusion, CYP3A5 was the single most important determinant of tacrolimus metabolite/parent ratios in a cohort of stable renal transplant recipients, in agreement with previous data. We demonstrated that CYP3A4 activity, assessed using the MDZ probe, bore little to no relationship with tacrolimus metabolite/parent ratios. In addition, these results suggest that 13-DMT and 31-DMT may display nonlinear elimination behavior during exposure to the combined CYP3A4/P-gp inhibitor itraconazole, possibly due to saturation of P-gp-dependent biliary efflux of metabolites and/or competition with tacrolimus for the catalytic site of CYP3A.

\section{Acknowledgments}

We thank study nurses J. De Vis, H. Wielandt, and V. Verbeeck. We also thank L. Baeté, M. Dekens, and J. Paulissen for technical assistance.

\section{Authorship Contributions}

Participated in research design: Vanhove, Annaert, Kuypers. Conducted experiments: Vanhove, de Jonge, de Loor, Oorts. Contributed new reagents or analytic tools: de Loor, Pohanka.

Performed data analysis: Vanhove. 
Wrote or contributed to the writing of the manuscript: Vanhove, de Jonge, de Hoon, Annaert, Kuypers.

\section{References}

Barrett JC, Fry B, Maller J, and Daly MJ (2005) Haploview: analysis and visualization of LD and haplotype maps. Bioinformatics 21:263-265.

Copeland KR, Thliveris JA, and Yatscoff RW (1990) Toxicity of cyclosporine metabolites. Ther Drug Monit 12:525-532.

de Jonge H, de Loor H, Verbeke K, Vanrenterghem Y, and Kuypers DR (2012) In vivo CYP3A4 activity, CYP3A5 genotype, and hematocrit predict tacrolimus dose requirements and clearance in renal transplant patients. Clin Pharmacol Ther 92:366-375.

de Jonge H, de Loor H, Verbeke K, Vanrenterghem Y, and Kuypers DRJ (2013) Impact of CYP3A5 genotype on tacrolimus versus midazolam clearance in renal transplant recipients: new insights in CYP3A5-mediated drug metabolism. Pharmacogenomics 14:1467-1480

de Loor H, de Jonge H, Verbeke K, Vanrenterghem Y, and Kuypers DR (2011) A highly sensitive liquid chromatography tandem mass spectrometry method for simultaneous quantification of midazolam, 1'-hydroxymidazolam and 4-hydroxymidazolam in human plasma. Biomed Chromatogr 25:1091-1098.

Gonschior AK, Christians U, Winkler M, Linck A, Baumann J, and Sewing KF (1996) Tacrolimus (FK506) metabolite patterns in blood from liver and kidney transplant patients. Clin Chem $\mathbf{4 2}$ 1426-1432.

Iwasaki K, Shiraga T, Matsuda H, Nagase K, Tokuma Y, Hata T, Fujii Y, Sakuma S, Fujitsu T, Fujikawa A, et al. (1995) Further metabolism of FK506 (tacrolimus). Identification and biological activities of the metabolites oxidized at multiple sites of FK506. Drug Metab Dispos 23: 28-34.

Kharasch ED, Walker A, Isoherranen N, Hoffer C, Sheffels P, Thummel K, Whittington D, and Ensign D (2007) Influence of CYP3A5 genotype on the pharmacokinetics and pharmacodynamics of the cytochrome P4503A probes alfentanil and midazolam. Clin Pharmacol Ther $\mathbf{8 2}$ $410-426$.

Lamba J, Hebert JM, Schuetz EG, Klein TE, and Altman RB (2012) PharmGKB summary: very important pharmacogene information for CYP3A5. Pharmacogenet Genomics 22:555-558.

Levey AS, Greene T, Schluchter MD, Cleary PA, Teschan PE, Lorenz RA, Molitch ME, Mitch WE, Siebert C, Hall PM, et al.; Modification of Diet in Renal Disease Study Group and the Diabetes Control and Complications Trial Research Group (1993) Glomerular filtration rate measurements in clinical trials. J Am Soc Nephrol 4:1159-1171.

Miller SA, Dykes DD, and Polesky HF (1988) A simple salting out procedure for extracting DNA from human nucleated cells. Nucleic Acids Res 16:1215.
Möller A, Iwasaki K, Kawamura A, Teramura Y, Shiraga T, Hata T, Schäfer A, and Undre NA (1999) The disposition of ${ }^{14} \mathrm{C}$-labeled tacrolimus after intravenous and oral administration in healthy human subjects. Drug Metab Dispos 27:633-636.

Radeke HH, Christians U, Bleck JS, Sewing KF, and Resch K (1991) Additive and synergistic effects of cyclosporine metabolites on glomerular mesangial cells. Kidney Int 39 $1255-1266$.

Shimomura M, Masuda S, Goto M, Katsura T, Kiuchi T, Ogura Y, Oike F, Takada Y, Uemoto S, and Inui K (2008) Required transient dose escalation of tacrolimus in living-donor liver transplant recipients with high concentrations of a minor metabolite M-II in bile. Drug Metab Pharmacokinet 23:313-317.

Stephens E, Bolderson I, Clark B, Kinsey S, Gooi HC, and Cook G (2006) The measurement of whole blood pre-treatment cyclosporine A: metabolite ratios predicts the onset of renal dysfunction in recipients of allogeneic stem cell transplantation. Ann Clin Biochem 43:382-388.

Stephens M, Smith NJ, and Donnelly P (2001) A new statistical method for haplotype reconstruction from population data. Am J Hum Genet 68:978-989.

Vanhove T, Annaert P, and Kuypers DRJ (2016) Clinical determinants of calcineurin inhibitor disposition: a mechanistic review. Drug Metab Rev 48:88-112.

Vanhove T, Annaert P, Lambrechts D, and Kuypers DRJ (2017a) Effect of ABCB1 diplotype on tacrolimus disposition in renal recipients depends on CYP3A5 and CYP3A4 genotype. Phar macogenomics $J$ 17:556-562.

Vanhove T, Bouillon T, de Loor H, Annaert P, and Kuypers D (2017b) Fexofenadine, a putative in vivo P-glycoprotein probe, fails to predict clearance of the substrate tacrolimus in renal recipients. Clin Pharmacol Ther 102:989-996.

Vermeer LMM, Isringhausen CD, Ogilvie BW, and Buckley DB (2016) Evaluation of ketoconazole and its alternative clinical CYP3A4/5 inhibitors as inhibitors of drug transporters: the in vitro effects of ketoconazole, ritonavir, clarithromycin, and itraconazole on 13 clinicallyrelevant drug transporters. Drug Metab Dispos 44:453-459.

Wang EJ, Lew K, Casciano CN, Clement RP, and Johnson WW (2002) Interaction of common azole antifungals with P glycoprotein. Antimicrob Agents Chemother 46:160-165.

Zheng S, Tasnif Y, Hebert MF, Davis CL, Shitara Y, Calamia JC, Lin YS, Shen DD, and Thummel KE (2012) Measurement and compartmental modeling of the effect of CYP3A5 gene variation on systemic and intrarenal tacrolimus disposition. Clin Pharmacol Ther 92:737-745.

Address correspondence to: Dr. Dirk Kuypers, Department of Nephrology and Renal Transplantation, University Hospitals Leuven, Herestraat 49, 3000 Leuven, Belgium. E-mail: dirk.kuypers@uzleuven.be 